Helgoländer wiss. Meeresunters. 30, 575-581 (1977)

\title{
Organisation in the pelagic ecosystem
}

\author{
T. Platt \& K. Denman \\ Marine Ecology Laboratory, Bedford Institute of Oceanography; \\ Dartmouth, Nova Scotia, Canada
}

\begin{abstract}
A continuous, steady-state theory has been developed for the abundance of organisms in the pelagic ecosystem as a function of their body weight. It is based on accepted relationships for the weight-dependence of metabolism and growth, in a context where individual organisms are assigned to one of a series of size classes for which the nominal weights increase in a geometric progression. Analysis of the biomass flow in such a representation leads to the conclusion that, in the steady state, the total biomass in any given size class decreases in a regular manner with increasing size. Explicitly, $b\left(w_{2}\right) / b\left(w_{1}\right) \sim\left(w_{2} / w_{1}\right)-0.22$, where $b\left(w_{2}\right)$ and $b\left(w_{1}\right)$ are the total biomasses in the size classes characterised by weights $w_{2}$ and $w_{1}$, respectively. The exponent $(-0.22)$ represents a balance between catabolism and anabolism, based on published reviews concerning the revelant parameters. This result agrees favourably with data collected by other workers in the subtropical oceans. The theory can be used to draw conclusions about the functional dynamics of the pelagic ecosystem, such as community respiration and rate of biomass flow.
\end{abstract}

\section{INTRODUCTION}

Many of the important processes in the lives of organisms are size-dependent; that is, their rate can be expressed as a simple function of the individual's body weight. In theoretical descriptions of physiological processes, it is therefore often convenient to use body weight as a scaling factor. It has long been known that these ideas could be applied to both the metabolism and growth of organisms, but the extreme generality of the relationships was, perhaps, not fully appreciated until the recent articles of synthesis by Dickie (1972), Fenchel (1974) and Banse (1976).

The universality of the relationships describing the size-dependence of metabolism and growth is so strong that one has been tempted to use them as the basis for a theory of the distribution of organisms by size in the pelagic food chain of the open ocean (Platt \& Denman, in press). Interest in such a theory arises from the fact that data on the size distribution of organisms in the sea can be collected automatically for the size range 1-100 $\mathrm{m}$ (Sheldon et al., 1972, 1973), and that instrument development is in progress to extend the size range over which continuous, automatic census can be made (Parsons \& Seki, 1969).

In this paper, we present an outline of the theory of size-distribution in the pelagic ecosystem, deduce some consequences relating to the flux of energy through 
the system and to the total community metabolism, and give a numerical example based on data from the Sargasso Sea.

\section{THEORY OF THE SIZE-DISTRIBUTION}

Let $\beta(w)$ be the total biomass $b(w)$ in the size-class characterised by weight $w$, divided by the width of the size-class $\triangle w$

$$
\beta(w)=\frac{b(w)}{\triangle w} .
$$

It is convenient (both mathematically, and in practical measurement) to arrange the size-classes in an increasing octave-scale. In other words, the characteristic weight of each size-class is double that of its smaller neighbour, and one half of that corresponding to its larger neighbour. At any instant in time, every organism in the system may be assigned (regardless of its species), uniquely, to one of the sizeclasses on this octave scale. With this arrangement of size-classes, the class widths, $\triangle w$, are, to within a constant factor of order unity, equal to the characteristic weight $w$

$$
\triangle w \simeq w
$$

Then the quantity $\beta(w)$ takes the form

$$
\beta(w) \simeq \frac{b(w)}{w} .
$$

We call $\beta(w)$ the normalised size-spectrum: it is an estimate of the number density of organisms in each class $w$. To find an expression for $\beta(w)$ we follow the flow of biomass (or energy) through the spectrum as it passes from smaller- to larger-sized particles. It is important to make a distinction between this kind of calculation, and a calculation following the growth of an individual particle.

We define $F(w)$ to be the steady-state flux of biomass leaving the spectral band characterised by mean weight $w$. In our simplified theory, we suppose $F(w)$ to be a one-way flux; that is, we assume the flow of energy is always from small-scale to largescale, rather than the reverse. One can think of cases where this will not be true, but in general the hypothesis of a unidirectional flux $F(w)$ does not conflict with accepted ideas on the structure of the pelagic ecosystem (e.g. Sheldon et al., 1973).

We can estimate the magnitude of $F(w)$ as the total biomass $b(w)$ in band $w$, divided by its turnover time $\tau(w)$

$$
F(w) \simeq \frac{b(w)}{\tau(w)} \simeq \frac{\beta(w) \cdot w}{\tau(w)} .
$$

Fenchel (1974) gives an empirical expression for the weight-dependence of turnover time:

$$
\tau(w) \sim A w^{x}
$$

where $A$ and $x$ are constants. Then, an expression for $F(w)$ takes the form

$$
F(w) \sim A^{-1} \beta(w) w^{1-x} .
$$


We now seek an expression for the rate of change of the flux with weight, $d F(w) / d w$. To find this we consider the possible ways in which biomass (or energy) is lost in the transformation of energy from smaller to larger sized particles. The two most important loss terms are likely to be first, metabolism; and second, the loss to the detritus food chain through unassimilated ration. A third possibility, which we shall not consider explicitly for the open ocean case, is the direct loss through sinking. Thus, we can write

$$
\frac{d F(w)}{d w} \sim-\left[\beta(w) \alpha \frac{w}{w}+q \frac{\beta(w)}{\tau(w)}\right] .
$$

This equation requires some explanation. The minus sign indicates that we are dealing with loss terms. The flux of energy is diminishing as it is transferred from smaller to larger particles. The first term on the right represents the loss due to metabolism. It is estimated from the number of particles respiring multiplied by their metabolism per unit weight, where we have used the well-known relationship, awr, for the weight dependence of metabolic rate (e.g. Fenchel, 1974). The second term on the right represents the loss to the decomposer food chain through unassimilated ration. It is estimated as a constant loss rate $q$ operating on the turnover of the individuals in a particular size class.

Eqn. (7) may be rewritten as follows:

$$
\frac{d F(w)}{d w} \sim-\left[\beta(w) \alpha w^{\gamma-1}+q \frac{F(w)}{w}\right] .
$$

If we substitute for $F(w)$ in eqn. (8) and set the result equal to the derivative of eqn. (6), we find

$$
\beta(w)(1-x) w^{-x}+w^{1-x} \frac{d \beta(w)}{d w} \sim-\left[A \beta(w) \alpha w^{-1}+q \beta(w) w^{-x}\right] .
$$

It is shown by Fenchel (1974) that, to a very good approximation, $\gamma+x \approx 1$. With this simplification, eqn. (9) becomes

$$
-\frac{d \beta(w)}{\beta} \sim[(1-x)+A \alpha+q] \frac{d w}{w},
$$

which may be integrated to yield:

$$
\frac{\beta(w)}{\beta_{o}} \sim\left(\frac{w}{w_{o}}\right)-(1-x+\alpha A+q),
$$

where the integration is from some band $\left(\beta_{0}, w_{0}\right)$, say, up to some arbitrary band $(\beta(w), w)$. Eqn. (11) represents the final, analytic result for the normalised biomass spectrum obtained by Platt $\&$ Denman (in press), where it is shown that the coefficient $q$ may be neglected compared to $(1-x+\alpha A)$. A simpler form of eqn. (11) is then

$$
\frac{\beta(w)}{\beta_{o}} \sim\left(\frac{w}{w_{o}}\right)^{-(1-x+\alpha A)} \text {. }
$$

Fenchel (1974) gives a value of $x$ (dimensionless) $=0.28$ with no apparent variation between unicells and heterotherms. The product $\alpha A$ (dimensionless) may 
be estimated from the data in Fenchel (1974) to be $\sim 0.5$ for heterotherms and $\sim 0.1$ for unicells. Taking, for the sake of a specific example, the data for heterotherms, we find the exponent in eqn. (12) to be $-(1-0.28+0.5)$ or -1.22 . For the purpose of comparing this result with data collected in the field, it is convenient to convert to a biomass spectrum $b(w)$, using eqn. (3). Then we have

$$
\frac{b(w)}{b_{0}} \sim\left(\frac{w}{w_{0}}\right)^{-0.22}
$$

On the assumption that the metabolic rate constants measured in the laboratory are representative of the performance of organisms in the wild, eqn. (13) implies that the biomass spectrum measured in the open ocean should have a slope, on a logarithmic plot, of about -0.22 throughout most of its range, but should be less negative at smaller scales because of the smaller value of $\alpha A$ for unicellular organisms. This result is in good agreement with the open ocean examples presented by Sheldon et al. (1972, 1973).

\section{THE BIOMASS FLUX $F(w)$}

Combining eqns. (6) and (11) gives us an expression for the biomass (energy) flux $F(w)$

$$
F(w) \sim A^{-1} \beta_{o}\left(\frac{w}{w_{0}}\right)^{-(1-x+\alpha A+q)} w^{1-x} .
$$

Using eqn. (6) we can get $\beta_{\circ}$ in the form

$$
\beta_{o} \sim A F\left(w_{0}\right) \cdot w_{0}^{(x-1)},
$$

whence (14) may be rewritten as

$$
F(w) \sim F\left(w_{0}\right)\left(\frac{w}{w_{0}}\right)-(\alpha A+q) .
$$

Let us now choose the reference size class $w_{0}$ to be the band such that $F\left(w_{0}\right)$ is equal to the net primary production $P_{n}$. Then we can write

$$
F(w) \sim P_{n}\left(\frac{w}{w_{p}}\right)^{-(\alpha A+q)}
$$

where $w_{p}$ now refers to the primary producers. Note that this approach to an expression for the biomass flux is oversimplified to the extent that in practice we expect the primary production to be spread over several size classes rather than concentrated in one single band. Nevertheless we expect that an equation analogous to eqn. (17), that is a flux decreasing roughly as $w^{-0.5}$, will hold for all size classes in the system larger than some upper limit beyond which there is no further autotrophic production. Based on eqn. (17) we may formulate an expression for the loss rate $d F(w) / d w$ as

$$
\frac{d F(w)}{d w} \sim-(\alpha A+q) \frac{P_{n}}{w_{p}}\left(\frac{w}{w_{p}}\right)^{-(1+\alpha A+q)}
$$




\section{TOTAL COMMUNITY METABOLISM}

Since $\beta(w)$ may be interpreted as a number density spectrum, we may calculate directly the total community metabolism $R$ for the pelagic ecosystem as

$$
R=\int \beta(w) \alpha w \gamma d w
$$

where the integral is taken over all size classes present in the system. Substituting for $\beta(w)$ from eqn. (11) we find

$$
R=\alpha \beta_{o} w_{0}(1-x+\alpha A+q) \int w-(1-x-y+\alpha A+q) d w .
$$

Using once more the simplification $\gamma+x \approx 1$ based on Fenchel's (1974) review of the published experimental data, we find

$$
R=\alpha \beta_{o} w_{0}^{(1-x+\alpha A+q)} \int w-(\alpha A+q) d w .
$$

Integration gives

$$
R=\frac{\alpha \beta_{o} w_{o}(1-x+\alpha A+q)}{1-\alpha A-q}[w(1-\alpha A-q)] \begin{gathered}
w_{f} \\
w_{o}
\end{gathered},
$$

where $w_{f}$ is the weight of the terminal predator in the system.

Now in the steady-state, the total community metabolism, $R$, should just balance the gross primary production $P_{g}$ plus the small remaining biomass flux $F\left(w_{i}\right)$. We may therefore write

$$
\begin{gathered}
P_{g}=\frac{\alpha \beta_{0} w_{o}^{(1-x+\alpha A+q)}}{1-\alpha A-q}\left[w_{f}(1-\alpha A-q)-w_{o}(1-\alpha A-q)\right] \\
+\frac{\beta_{o} w_{0}^{(1-x)}}{A}\left(\frac{w_{f}}{w_{o}}\right)^{-(\alpha A+q)}
\end{gathered}
$$

where eqn. (14) has been substituted for $F(w)$. In practice, the second term in the square bracket (lower limit of integration) will be negligible compared to the first, such that

$$
P_{g} \approx \frac{\alpha \beta_{o} w_{0}^{(1-x)}}{1-\alpha A-q}\left(\frac{w_{f}}{w_{0}}\right)^{-(\alpha A+q)}\left[w_{f}+\frac{1-\alpha A-q}{\alpha A}\right]
$$

The second term in the square bracket will again usually be negligible, such that

$$
P_{g} \approx \frac{a \beta_{o} w_{o}(1-x+\alpha A+q)}{1-\alpha A-q} w_{f}(1-a A-q) .
$$

Notice that in this calculation it matters not whether the primary production is concentrated in one band or spread over several bands. It is simply a consequence of the steady-state requirement that the total primary production should equal the total system metabolism.

Another point concerning eqn. (25) is that it is dimensionally inconsistent: the units of $P_{g}$ are $\mathrm{gm}^{-3} \mathrm{~s}^{-1}$, and of the right hand side $\mathrm{m}^{-3} \mathrm{~s}^{-1}$. This inconsistency results from the integration (19) over $w$, a quantity which occurs in the equations raised to fractional powers and which had previously been treated as dimensionless. The problem is avoided if a dimensionless weight $w^{\prime}=w / w_{0}$ is used in the equations from the beginning. Such a procedure is outlined in the Appendix. 


\section{A NUMERICAL EXAMPLE}

Let us evaluate eqn. (25) for data collected in the Sargasso Sea, a location for which the assumption of steady-state should not be far wrong. We have used the data tabulated in Strickland (1960), where the entries for the Sargasso Sea are based mainly on the work of Riley. We take $\alpha$ to be $2 \times 10^{-7} \mathrm{~s}^{-1}$ and the product $\alpha A$ to be 0.5, following Fenchel (1974), and we set $q=0$ for the reasons given in Platt $\&$ Denman (in press). For $w_{\mathrm{f}}$ we take a value $10^{5} \mathrm{~g}$, corresponding to the larger tuna species, and for $w_{0}$ a value of $10^{-8} \mathrm{~g}$, corresponding roughly to a cell $20 \mu \mathrm{m}$ in diameter. Strickland gives values for the primary production of about $1 \mathrm{mg} \mathrm{C} \mathrm{m}{ }^{-3}$ $\mathrm{day}^{-1}$, roughly equal to $10^{-8} \mathrm{~g} \mathrm{~m}^{-3} \mathrm{~s}^{-1}$. Substituting these values into eqn. (25) and solving for $\beta_{0}$ gives an estimate for $\beta_{0}$ of $0.5 \times 10^{6} \mathrm{~m}^{-3}$, equivalent to a biomass $b_{o}$ of $5 \mathrm{mg} \mathrm{m}^{-3}$. The tabulation in Strickland (1960) indicates from 1 to $3 \mathrm{mg} \mathrm{C} \mathrm{m}^{-3}$ for the particulate carbon concentration measured by conventional methods. This crude calculation gives some measure of the internal consistency of the theory of organisation of the pelagic ecosystem.

\section{APPENDIX}

Equations for size-dependent growth or metabolism often contain the weight $w$ of the organism raised to a non-integral power. Usually, it is sufficient to associate the dimensions of the expression solely with the proportionality constant. However, if an expression (which does not contain $d w$ ) is integrated over $w$ as was done in eqn. (19), a dimensional inconsistency will result. Such a problem can be avoided by the use of nondimensional variables.

Let us define a nondimensional weight $w^{\prime}=w_{/} w_{0}$. The expression for the rate of metabolism per organism (units $\mathrm{s}^{-1}$ ) becomes

$$
\begin{aligned}
M & =\alpha w^{\gamma} \\
& =\alpha^{\prime} w^{\prime} \gamma \\
\text { where } \quad \alpha^{\prime} & =\alpha w_{0}^{\gamma} \text { with units s }{ }^{-1} .
\end{aligned}
$$

Equation (19) becomes

$$
\begin{gathered}
R=\int_{w_{0}}^{w_{f}} \beta(w) \alpha w y d w \\
=\int_{1}^{w_{f}^{\prime}} \alpha^{\prime} w_{o} \beta_{o} w^{\prime}-(1-x+\alpha A+q) w^{\prime} \gamma d w^{\prime} \\
=w_{0} \alpha^{\prime} \beta_{o} \int_{1}^{w_{f}^{\prime}} w^{\prime}-(\alpha A+q) d w^{\prime} \\
=\frac{w_{o} \alpha^{\prime} \beta_{o}}{1-\alpha A-q}\left[w_{f}^{\prime}-(\alpha A+q-1)-1\right]
\end{gathered}
$$

which is the equation analogous to (22). The units of $R$ are $\mathrm{gm}^{-3} \mathrm{~s}^{-1}$ and the units 
of the right hand side are just the units of $w_{o} \alpha^{\prime} \beta_{o}$ which are $\mathrm{gs}^{-1} \mathrm{~m}^{-3}$. The dimensions are now consistent.

\section{LITERATURE CITED}

Banse, K., 1976. Rates of growth, respiration and photosynthesis of unicellular algae as related to cellsize - a review. J. Phycol. 12, 135-140.

Dickie, L. M., 1972. Food chains and fish production. ICNAF Spec. Publ. 8, 201-221.

Fenchel, T., 1974. Intrinsic rate of natural increase: the relationship with body size. Oecologia $14,317-326$.

Leim, A. H. \& Scott, W. B., 1966. Fishes of the Atlantic coast of Canada, J. C. Stevenson (Ed.). Bull. Fish. Res. Bd Can. 155, 288-291.

Parsons, T. R. \& Seki, H., 1969. A short review of some automated techniques for the detection and characterization of particles in sea water. Bull. Jap. Soc. Fish. Oceanogr. Spec. Nr. (Prof. Uda's Commemorative Papers), 173-177.

Platt, T. \& Denman, K. L. The structure of pelagic marine ecosystems. Rapp. P.-v. Réun. Cons. int. Explor. Mer. (In press).

Sheldon, R. W., Prakash, A. \& Sutcliffe, W. H., 1972. The size distribution of particles in the ocean. Limnol. Oceanogr. 17, 327-340.

- Sutcliffe, W. H. \& Prakash, A., 1973. The production of particles in the surface waters of the ocean with particular reference to the Sargasso Sea. Limnol. Oceanogr. 18, 719-733.

Strickland, J. D. H., 1960. Measuring the production of marine phytoplankton. Bull. Fish. Res. Bd Can. 122, 172 p.

First author's address: Dr. T. Platt

Marine Ecology Laboratory

Bedford Institute of Oceanography

Dartmouth, N.S. B2Y 4A2

Canada 